\title{
Evaluación técnica de albañilería de bloques con adiciones puzolánicas
}

\section{Technical evaluation of block masonry with pozzolanic additions}

Fecha de entrega: 23 de mayo 2018 Fecha de aceptación: 8 de octubre 2018

\section{Walter Roldán ${ }^{1}$ y Julio Soto ${ }^{2}$}

\author{
${ }^{1}$ Departamento de Ingeniería Civil, Universidad Católica del Norte, Av. Angamos 0610, Antofagasta, Chile, wroldan@ucn.cl \\ ${ }^{2}$ Laboratorio Liemun, Universidad Católica del Norte, Radomiro Tomic 8095, Antofagasta, Chile, jotasotolimari@gmail.com
}

Se efectúan ensayos de resistencia mecánica a diversas probetas de albañilería de bloques de hormigón, reemplazando cemento Portland por puzolanas disponibles en la región de Antofagasta, Chile (ceniza volante, ceniza volcánica y polvo del desierto). Para caracterizar la resistencia de la albañilería, se ensayan a compresión bloques individuales, prismas y muretes, fabricados con hormigón con distintas combinaciones conglomerantes del tipo cemento-puzolana; las resistencias obtenidas se comparan con una mezcla patrón con $100 \%$ de cemento. Los resultados indican que es factible reemplazar un porcentaje de cemento Portland por puzolanas, obteniéndose resistencias muy similares, contribuyendo con ello a reducir costos de construcción y disminuyendo las emisiones de gases de efecto invernadero que se generan en la calcinación de la materia prima. Se pretende también realzar la importancia de la albañilería de bloques de hormigón en la edificación de viviendas de bajo costo y amigables con el ambiente.

Palabras clave: albañilería, bloques de hormigón, cemento puzolánico, construcción verde

\section{Introducción}

Dada la carencia de arcillas en la zona norte de Chile, la construcción con albañilería de bloques para viviendas de mediana y baja altura es una opción muy recurrida debido al bajo costo en comparación al hormigón armado (Roldán, 2003). A su vez, la fabricación de bloques de hormigón con remplazo parcial de cemento por puzolana, es una opción que tiene ventajas económicas por cuanto las puzolanas están disponibles a un menor costo que el cemento, tiene ventajas tecnológicas porque se pueden disponer de buenos valores de resistencia mecánica y de mejores condiciones de durabilidad (Metha y Monteiro, 2006; Malhotra y Metha 1996). No se puede dejar de considerar que también tiene beneficios de sostenibilidad ambiental porque al reducir la demanda de cemento
Tests are carried out on various specimens of concrete block masonry, replacing Portland cement with pozzolans available in the region of Antofagasta, Chile (fly ash, volcanic ash and desert dust). To characterize the strength of the masonry, individual blocks, prisms and small walls are tested, made of concrete with different binder combinations «cement-pozzolan»; the obtained resistances are compared with standard mixture (100\% cement). The results indicate that it is feasible to replace a percentage of Portland cement with pozzolans, obtaining very similar resistance, contributing to reduce construction costs and also, contributing to the environment by reducing cement demand and thereby reducing emissions of greenhouse gases that are generated in the calcination of the raw material. It is also pretension to enhance the importance of the masonry of concrete blocks in the construction of low-cost housing (economic and ecological).

Keywords: masonry, concrete blocks, pozzolanic cement, green construction

disminuyen las emisiones de $\mathrm{CO}_{2}$ a la atmósfera (O'Rourke et al., 2009). Se disponen de investigaciones previas con uso de materiales puzolánicos en la región de Antofagasta (Roldán et al., 2013; Roldán y Pavéz, 2011; Roldán y Robles, 2014), los que muestran que la ceniza volcánica (puzolana natural) posee buenas propiedades cementantes, pero de reactividad levemente inferior a la ceniza volante producida en planta termoeléctrica; a su vez, la reactividad puzolánica del polvo del desierto, es menor a la ceniza volcánica.

Es por ello que se busca aplicar nuevos conglomerantes del tipo Cemento Portland/Puzolana (Metha y Monteiro, 2006), que reflejen la reactividad puzolánica detectada en estudios previos realizados en la región de Antofagasta, ya citados. Por ello, se plantea elaborar unidades de 
albañilería de bloques microvibrados con las siguientes combinaciones conglomerantes (en peso): Cemento/ Ceniza Volante $=60 / 40$, Cemento/Ceniza Volcánica 70/30, Cemento/Polvo del Desierto $=80 / 20$ y también $100 \%$ Cemento en el conglomerante, cuyos resultados servirán de comparación o control; estimándose además índices de costos asociados a cada producto obtenido.

\section{Caracterización de materiales}

\section{Características del cemento}

Se utiliza cemento Portland de alta resistencia OPC, sin adiciones puzolánicas. En Tabla 1 se presenta su composición mineralógica obtenida de ensayos de fluorescencia de rayos $x(x R F)$.

\section{Puzolanas}

\section{Ceniza volante}

Polvo fino originado por la combustión de carbón mineral en la planta termoeléctrica de la ciudad de Tocopilla, Chile. El resultado de análisis químico xRF se presenta en Tabla 1.

\section{Ceniza volcánica}

Polvo fino resultante de erupciones volcánicas luego de expulsar a la atmósfera ceniza en estado incandescente, que al decantar forma depósitos en diversos lugares; muchos de ellos a grandes distancias de su fuente de origen y que permanecen ahí desde el Mioceno (Breitkreuz et al., 2014). La ceniza volcánica utilizada proviene de depósito natural ubicado en la proximidad del $\mathrm{km} 1304$ en la Ruta 5 Norte, en la región de Antofagasta, Chile. El resultado de análisis químico xRF se presenta en Tabla 1.

\section{Polvo del desierto}

Es un material natural, suelto, disperso, finamente pulverizado, de color ocre que otorga el color característico al desierto del norte de Chile; de manera coloquial se le denomina con el nombre de chuca, chusca o chuzca. Se encuentra en depósitos naturales, dispuesto en espesores superficiales de suelo de 10 a $30 \mathrm{~cm}$. Es un material pulverulento formado por mezclas de diversos componentes: suelos de origen volcánico, arcilla, yeso, fragmentos de roca pulverizada y materiales diversos típicos del desierto, los que han estado expuestos por largos años a la radiación solar. El material utilizado proviene de depósitos de libre acceso ubicados en la proximidad del km 1385, en la Ruta 5 Norte, región de Antofagasta, Chile. Se trabaja con material natural bajo malla \#100 ASTM, el que resulta con una distribución granulométrica que se caracteriza por un tamaño medio de $47.1 \mu \mathrm{m}$, con $90 \%$ bajo $130.1 \mu \mathrm{m}$, con $50 \%$ bajo $20.6 \mu \mathrm{m}$ y con $10 \%$ bajo 1.4 $\mu \mathrm{m}$. Posee una densidad real de $2.23 \mathrm{~g} / \mathrm{cm}^{3}$. Los resultados del análisis químico xRF se presentan en Tabla 1, los cuales fueron obtenidos en el Laboratorio de Geoquímica Aplicada de la Universidad Católica del Norte.

Tabla 1: Composición química de materiales cementantes utilizados de ensayos xRF

\begin{tabular}{|l|c|c|c|c|}
\hline Compuesto, \% & $\begin{array}{c}\text { Cemento } \\
\text { OPC }\end{array}$ & $\begin{array}{c}\text { Ceniza } \\
\text { volante }\end{array}$ & $\begin{array}{c}\text { Ceniza } \\
\text { volcánica }\end{array}$ & $\begin{array}{c}\text { Polvo } \\
\text { desierto }\end{array}$ \\
\hline $\mathrm{SiO}_{2}$ & 15.1 & 54.8 & 63.6 & 46.7 \\
\hline $\mathrm{Al}_{2} \mathrm{O}_{3}$ & 4.4 & 21.2 & 12.7 & 12.8 \\
\hline $\mathrm{Fe}_{2} \mathrm{O}_{3}$ & 3.4 & 7.5 & 2.8 & 3.8 \\
\hline $\mathrm{CaO}$ & 67.6 & 3.9 & 5.2 & 8.6 \\
\hline $\mathrm{MgO}$ & 0.6 & 2.7 & 3.1 & 4.5 \\
\hline $\mathrm{K}_{2} \mathrm{O}$ & 0.8 & 2.0 & 3.7 & 2.1 \\
\hline $\mathrm{Na}_{2} \mathrm{O}$ & 0.4 & 1.4 & 3.3 & 3.6 \\
\hline $\mathrm{SO}_{3}$ & 3.5 & 0.3 & 1.1 & 3.8 \\
\hline $\mathrm{Ppc}^{2}$ & 3.5 & 3.3 & 3.7 & 5.4 \\
\hline Otros & 0.7 & 2.4 & 0.3 & 7.2 \\
\hline $\mathrm{H}_{2} \mathrm{O}$ & 0.0 & 0.5 & 0.5 & 1.5 \\
\hline
\end{tabular}

Ppc: pérdida por calcinación; Otros: compuestos diversos $(\mathrm{Sr}, \mathrm{Ni}, \mathrm{Cu}$, $\mathrm{Zr}, \mathrm{Cr}, \mathrm{Zn}, \mathrm{V}, \mathrm{Rb}, \mathrm{Cl}, \mathrm{I}, \mathrm{Ba}, \mathrm{Mo}, \mathrm{Se})$

Tabla 2: Granulometría y propiedades de los áridos

\begin{tabular}{|c|c|c|c|}
\hline \multicolumn{2}{|c|}{ Tamices malla } & \multicolumn{2}{c|}{$\%$ que pasa } \\
\hline Malla N ${ }^{\circ}$ & Abertura, $\mathrm{mm}$ & Arena & Gravilla \\
\hline $1 / 2 "$ & 12.5 & 100 & 99 \\
\hline $3 / 8 ”$ & 10.0 & 100 & 92 \\
\hline$\# 4$ & 5.0 & 100 & 29 \\
\hline$\# 8$ & 2.5 & 90 & 2 \\
\hline$\# 16$ & 1.250 & 70 & 1 \\
\hline$\# 30$ & 0.630 & 46 & 1 \\
\hline$\# 50$ & 0.315 & 27 & 1 \\
\hline$\# 100$ & 0.160 & 16 & 1 \\
\hline Densidad real ${ }^{\mathrm{a}}, \mathrm{kg} / \mathrm{m}^{3}$ & 2580 & 2626 \\
\hline Densidad ap. suelta ${ }^{\mathrm{b}}, \mathrm{kg} / \mathrm{m}^{3}$ & 1570 & 1595 \\
\hline Densidad ap. compacta ${ }^{\mathrm{b}}, \mathrm{kg} / \mathrm{m}^{3}$ & 1720 & 1845 \\
\hline Absorción, \% & 0.74 & 0.80 \\
\hline
\end{tabular}

${ }^{\mathrm{a} N C h} 1239$ (2009) o NCh1117 (2010), ${ }^{\mathrm{N} C h} 1116$ (2008) 


\section{Áridos utilizados, arena y gravilla}

Para la elaboración de mezclas de hormigón para fabricar bloques y disponer de mortero de junta, se utilizó áridos de la zona, con características y propiedades indicadas en Tabla 2 .

\section{Fabricación de bloques de hormigón}

Para disponer de condiciones controladas en la fabricación de bloques, se procede a desarrollar el ciclo completo de fabricación en el laboratorio, diseñando y fabricando un molde metálico, el cual es mostrado en la Figura 1, con el cual se procede a fabricar bloques de hormigón con distintas combinaciones conglomerantes. Para cada combinación se produce la cantidad necesaria de bloques para ensayar probetas tanto de bloques individuales, prismas y muretes (5 probetas de cada tipo).

El molde metálico utilizado está conformado por tres partes desmontables: pared perimetral rectangular, responsable de las caras planas exteriores, definiendo las dimensiones del bloque según: ancho-largo-alto $=14-39-19 \mathrm{~cm}$; doble pieza tronco-piramidal central, que permite generar los huecos del bloque; y finalmente, la base metálica que permite unir y rigidizar todo el sistema de moldaje.
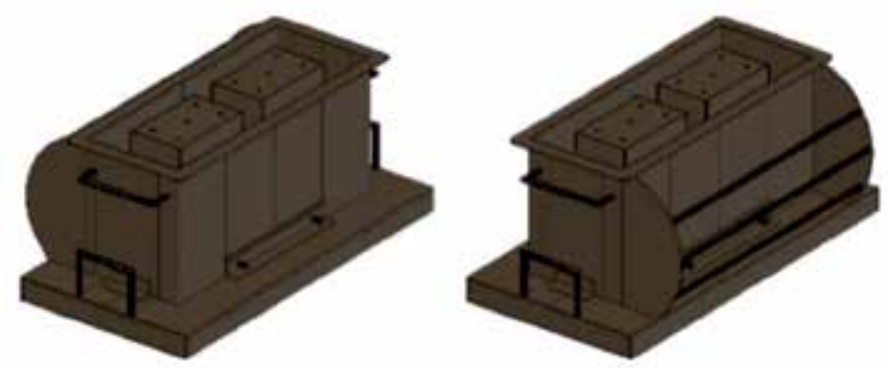

Figura 1: Molde metálico para fabricar bloques, vistas tridimensionales

El hormigón se produce en una betonera de 150 lt de capacidad y los bloques manualmente; la compactación de la mezcla se realiza con auxilio de una plataforma vibradora con motor eléctrico y eje con masa excéntrica. Una vez compactada la mezcla, el molde se voltea para dejar depositado el bloque en el piso de sala de curado en donde se mantienen a temperatura ambiente entre 23 y $25^{\circ} \mathrm{C}$ y humedad relativa ambiental comprendida entre 90 y $100 \%$ hasta la edad de ensayo.

\section{Dosificación de mezclas de hormigón utilizadas}

Se utilizan dosificaciones según combinación conglomerante descritas anteriormente y según las siguientes proporciones (en peso): razón agua/ conglomerante $=0.65$ y razón gravilla/arena $=1.0$.

Tabla 3: Dosificación para $1 \mathrm{~m}^{3}$ de hormigón para elaborar bloques

\begin{tabular}{|c|c|c|c|c|c|}
\hline & \multirow[b]{2}{*}{ Materiales } & \multicolumn{4}{|c|}{ Hormigón según conglomerante } \\
\hline & & $\begin{array}{l}100 \% \\
\text { OPC }\end{array}$ & $60 / 40$ & $70 / 30$ & $80 / 20$ \\
\hline \multirow{4}{*}{ 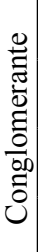 } & Cemento, $\mathrm{kg}$ & 277.0 & 166.2 & 193.9 & 221.6 \\
\hline & Ceniza volante, $\mathrm{kg}$ & - & 110.8 & - & - \\
\hline & Ceniza volcánica, $\mathrm{kg}$ & - & - & 83.1 & - \\
\hline & Polvo desierto, $\mathrm{kg}$ & - & - & - & 55.4 \\
\hline \multicolumn{2}{|c|}{ Agua, lt } & 180.0 & 180.0 & 180.0 & 180.0 \\
\hline \multicolumn{2}{|c|}{ Arena, $\mathrm{kg}$} & 897.4 & 897.4 & 897.4 & 897.4 \\
\hline \multicolumn{2}{|c|}{ Gravilla, $\mathrm{kg}$} & 897.4 & 897.4 & 897.4 & 897.4 \\
\hline
\end{tabular}

\section{Preparación de ensayos de unidades de albañilería de} bloques

Para el ensayo a compresión, se procede a refrentar las caras superior e inferior del bloque con mortero plástico compuesto de cemento Portland y yeso en partes iguales, de espesor no superior a $3 \mathrm{~mm}$ con el objeto de obtener paralelismo entre las dos superficies de carga, siguiendo indicaciones de la norma NCh1928 (1993). Un bloque preparado para ensaye se ilustra en Figura 2(a). La resistencia a la compresión de unidades de bloques quedará expresada como el cociente entre la carga de rotura por compresión y el área transversal a la aplicación de la carga. Una serie de cinco bloques se calculará sobre la base del área total de la unidad, incluido los huecos (área bruta), según lo indica la norma NCh1928 (1993); concepto de área bruta que se mantiene vigente para el cálculo de resistencia prismática. También, para adecuarse a las indicaciones de la norma NCh182 (2008), se prepara y ensaya otra serie de cinco bloques cuya resistencia a compresión se calculará sobre la base del área neta promedio, lo que permitirá comparar resultados de resistencia con el requisito de umbral mínimo promedio de $13 \mathrm{MPa}$ que señala la norma NCh181 (2006). 


\section{Preparación para ensayo de prismas de albañilería}

Se preparan cinco prismas de 3 hiladas de bloques cada uno, unidos entre sí por mortero de junta de la misma combinación conglomerante que el hormigón de bloques, de espesor aproximado de $10 \mathrm{~mm}$. Los prismas se ensayan con las caras superior e inferior adecuadamente refrentadas con pasta de yeso-cemento-agua, a la edad de 28 días desde su elaboración. Un prisma en proceso de preparación para ensayo se ilustra en Figura 2(b). La resistencia a compresión de prismas quedará expresada como el cociente entre la carga de rotura y el área bruta de la sección transversal, de acuerdo con indicaciones de la norma NCh1928 (1993).

\section{Preparación para ensayo de muretes de albañilería}

Se fabrican cinco muretes cuadrados de $60 \times 60 \mathrm{~cm}$ cada uno, lo que se materializa con hiladas de 1 bloque y medio en sentido horizontal y tres hiladas en sentido vertical, unidos por mortero de junta de la misma combinación que el hormigón de bloques. Transcurridos 28 días desde su elaboración, los muretes son ensayados aplicando carga de compresión en dirección de una de las diagonales principales con auxilio de cabezales metálicos apoyados en los vértices adecuadamente refrentados con pasta de yeso-cemento. Un murete preparado para ensayo se ilustra en Figura 2(c). En este caso, la resistencia mecánica se expresará como resistencia de corte según el cociente entre la carga máxima de rotura y el área que compromete la longitud de la diagonal principal y el espesor del murete, sin descontar la superficie de huecos de los bloques, según procedimiento descrito en norma NCh2123 (1997).

\section{Preparación para ensayo de mortero de junta}

El mortero de junta utilizado para la confección de prismas y muretes está conformado por la razón arena/ conglomerante/agua $=8 / 2 / 1$ (en peso); dosificación que se mantendrá fija tanto para el ensayo de prismas, como para el de muretes. Como material conglomerante se entiende la adición conjunta de cemento y puzolana en las combinaciones anteriormente descritas. Para el control de calidad del mortero se confeccionan probetas Rilem de $40 \mathrm{x}$ 40 x 160 mm que se ensayan a flexotracción y compresión, según indicaciones de la norma NCh158 (1967). El ensayo se realiza a edad de 28 días de curado sumergido en agua.

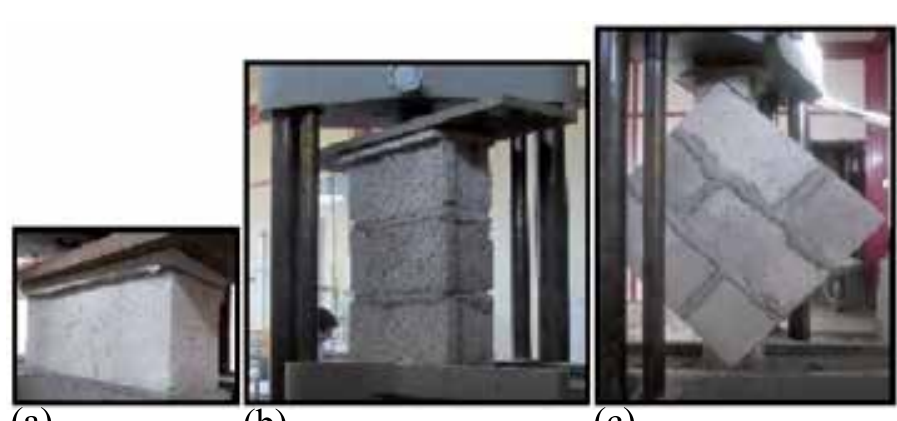

(a)

(b)

(c)

Figura 2: Disposición en la prensa de ensayo de probetas de albañilería de bloques de hormigón: a) unidad de albañilería, b) prisma de albañilería y c) murete de albañilería

\section{Determinación de la resistencia característica de compresión de probetas de albañilería}

Todos los valores de resistencia característica de probetas de albañilería se expresarán siguiendo indicaciones del Anexo A de NCh1928 (1993). Para obtener la resistencia característica, se debe cumplir con una edad de 28 días y ensayar 5 probetas en su tipo, donde el valor queda expresado por la siguiente ecuación:

$R_{k}=\bar{G}-0.431\left(G_{5}-G_{1}\right)$

donde $R_{k}$ es la resistencia característica correspondiente al tipo de probeta, $\bar{G}$ es la resistencia promedio de las cinco probetas ensayadas y $G_{5}, G_{1}$ corresponden al mayor y menor valor de resistencia obtenido de los ensayos, respectivamente. Por ello, se definen las siguientes resistencias características, dependiendo del tipo de probeta: a) resistencia característica de unidades de bloques de hormigón $f_{p}, \mathrm{~b}$ ) resistencia característica de prismas o resistencia prismática de bloques de hormigón $f_{\mathrm{m}}$ ' y c) resistencia característica de corte o resistencia básica de corte de muretes de bloques de hormigón $\tau_{\mathrm{m}}$.

\section{Criterio de selectividad de mezclas conglomerantes puzolánicas para fabricar bloques}

Se propone utilizar tres diferentes adiciones puzolánicas, en tres diferentes reemplazos de cemento Portland. En general, el parámetro que más incide en la decisión de selectividad de los materiales a utilizar es el resultado de la resistencia mecánica que se obtiene; sin embrago, también 
tiene incidencia en esta decisión el costo económico de su uso. Para el caso, es interesante comparar el aporte combinado de cada una de las mezclas conglomerantes a la intención de disponer de buena resistencia mecánica al más bajo costo económico. En tal sentido, se propone evaluar cada una de estas mezclas a través de la definición del parámetro arbitrario $K$, que representa el valor cociente entre la resistencia mecánica y el costo económico.

$$
\nVdash=\frac{\text { Resistencia mecánica }}{\text { Costo económico }}
$$

A través del parámetro $K$, se discriminará en la búsqueda de la mezcla más conveniente según el mayor valor que resulte en su evaluación; considerando en el numerador la resistencia prismática $f_{\mathrm{m}}$, (o la resistencia básica de corte $\left.\tau_{\mathrm{m}}\right)$ y en el denominador, el costo económico del conglomerante. Para determinar el costo económico del conglomerante, se asume que el costo económico referencial de adquisición de una unidad de peso de cemento Portland (por ejemplo, tonelada), es equivalente al valor $100 \%$. También se puede estimar el costo económico equivalente de materiales puzolánicos, para la misma unidad de peso, asociándolo al costo económico de acopio, carguío y transporte hasta el centro de consumo y compararlo con el costo del cemento, resultando los siguientes costos económicos. Valor $18 \%$ para la ceniza volante, valor $14 \%$ para la ceniza volcánica y valor $10 \%$ para el polvo del desierto. Los materiales puzolánicos probados tienen un costo de adquisición menor que el cemento ya que en algunos casos son pasivos industriales que se desechan en botaderos como la ceniza volante. En otros casos se encuentran en depósitos naturales de libre disposición como la ceniza volcánica y el polvo del desierto. De este modo, el costo económico de los nuevos conglomerantes puzolánicos se determina de acuerdo al cálculo ponderado siguiente:

- Costo conglomerante ceniza volante 60/40:

$60 \%$ cemento $+40 \%$ ceniza volante $=0.60 \cdot 100 \%+$ $0.40 \cdot 18 \%=67.2 \%$

- Costo conglomerante ceniza volcánica 70/30:

$70 \%$ cemento $+30 \%$ ceniza volcánica $=0.70 \cdot 100 \%$

$+0.30 \cdot 14 \%=74.2 \%$

- Costo conglomerante polvo desierto 80/20:
$80 \%$ cemento $+20 \%$ polvo desierto $=0.80 \cdot 100 \%+$ $0.20 \cdot 10 \%=82.0 \%$

Es decir, con estos reemplazos se puede ahorrar entre 33\% y $18 \%$ del costo de utilizar conglomerante $100 \%$ cemento Portland en la elaboración de hormigón para bloques; cifras que describen un ahorro económico interesante, aunque para disponer de estas economías también es importante disponer de una adecuada resistencia mecánica. Es lo que también se pretende evaluar en esta investigación.

\section{Resistencia de probetas de albañilería de bloques de hormigón y mortero}

La Tabla 4 muestra los resultados experimentales de resistencia máxima, resistencia mínima, resistencia media y resistencia característica obtenidos en series de probetas de albañilería de bloques de hormigón elaboradas con distintas mezclas conglomerantes y mortero de junta. Para el caso de probetas de unidades de bloques, se muestran resistencias de serie referida al área bruta total (largo y ancho), sin descontar huecos; también, resultados de resistencias de serie referida al área neta promedio, calculada de acuerdo con indicaciones de la norma NCh182 (2008).

\section{Análisis de resultados de resistencia en bloques}

Para las diversas combinaciones conglomerantes, los resultados de resistencia a compresión en bloques referidos al área bruta y área neta, no presentan grandes variaciones entre sí, lo que indicaría que las mezclas y la fabricación de bloques han resultado homogéneas. Tampoco se observan diferencias importantes entre combinaciones conglomerantes con adiciones puzolánicas lo que viene a comprobar la reactividad puzolánica entre las distintas adiciones de reemplazo de cemento, coincidiendo con lo reportado por Roldán (2003). Se destaca que todas las unidades ensayadas superaron significativamente los requerimientos de resistencia que se exige al bloque como unidad de albañilería. Las normas chilenas de albañilería NCh1928 (1993) y NCh2123 (1997), indican que los bloques deben satisfacer los requisitos de la clase A especificados en la antigua norma NCh181 (1965), 4.5 MPa referidos al área bruta. Sin embargo, dicha norma no está vigente y ha sido reemplazada por la norma NCh181 (2006), la que especifica que el promedio de las unidades 
debe superar $13 \mathrm{MPa}$ y el bloque individual superar 12 $\mathrm{MPa}$; ambos valores medidos sobre el área neta. De la Tabla 4 es posible observar que, en todos los casos, los resultados de resistencia de unidades de bloque referidos al área bruta superan en por lo menos $200 \%$ la exigencia de $4.5 \mathrm{MPa}$ para la unidad y que cuando la exigencia cambia a $13 \mathrm{MPa}$ referidas al área neta, la superan en por lo menos $70 \%$. Esto demuestra que se están obteniendo buenos resultados de resistencia mecánica producto de los hormigones.

Tabla 4: Resistencia de probetas de albañilería de bloques de hormigón y mortero de junta

\begin{tabular}{|c|c|c|c|c|c|}
\hline \multirow{2}{*}{$\begin{array}{l}\text { Tipo de } \\
\text { probeta }\end{array}$} & \multirow{2}{*}{ Propiedades } & \multicolumn{4}{|c|}{ Hormigón según conglomerante } \\
\hline & & $\begin{array}{l}100 \% \\
\text { OPC }\end{array}$ & $60 / 40$ & $70 / 30$ & $80 / 20$ \\
\hline \multirow{5}{*}{$\begin{array}{c}\text { Bloques, área } \\
\text { bruta }\end{array}$} & Área bruta, $\mathrm{m}^{2}$ & 0.0557 & 0.0555 & 0.0557 & 0.0551 \\
\hline & $f_{c}$ máxima, $\mathrm{MPa}$ & 17.30 & 14.48 & 13.64 & 14.29 \\
\hline & $f_{c}$ mínima, $\mathrm{MPa}$ & 16.00 & 12.16 & 13.14 & 12.89 \\
\hline & $f_{c}$ promedio, $\mathrm{MPa}$ & 16.68 & 13.62 & 13.37 & 13.44 \\
\hline & $f_{p}, \mathrm{MPa}$ & 16.12 & 12.62 & 13.15 & 12.84 \\
\hline \multirow{5}{*}{$\begin{array}{c}\text { Bloques, área } \\
\text { neta }\end{array}$} & Área neta, $\mathrm{m}^{2}$ & 0.0329 & 0.0329 & 0.0331 & 0.0336 \\
\hline & $f_{c}$ máxima, $\mathrm{MPa}$ & 27.82 & 23.80 & 22.46 & 23.64 \\
\hline & $f_{c}$ mínima, $\mathrm{MPa}$ & 25.73 & 19.98 & 21.63 & 21.33 \\
\hline & $f_{c}$ promedio, $\mathrm{MPa}$ & 26.82 & 22.39 & 22.01 & 22.23 \\
\hline & $f_{p}, \mathrm{MPa}$ & 25.92 & 20.74 & 21.65 & 21.23 \\
\hline \multirow{4}{*}{ Prismas } & $f_{c}$ máxima, $\mathrm{MPa}$ & 5.01 & 4.34 & 4.52 & 4.78 \\
\hline & $f_{c}$ mínima, $\mathrm{MPa}$ & 4.37 & 4.25 & 3.99 & 4.24 \\
\hline & $f_{c}$ promedio, $\mathrm{MPa}$ & 4.62 & 4.28 & 4.16 & 4.42 \\
\hline & $f_{m}, \mathrm{MPa}$ & 4.34 & 4.25 & 3.93 & 4.19 \\
\hline \multirow{4}{*}{ Muretes } & $\tau$ máxima, $\mathrm{MPa}$ & 1.06 & 1.02 & 0.97 & 0.94 \\
\hline & $\tau$ mínima, $\mathrm{MPa}$ & 0.79 & 0.52 & 0.68 & 0.73 \\
\hline & $\tau$ promedio, $\mathrm{MPa}$ & 0.92 & 0.74 & 0.81 & 0.87 \\
\hline & $\tau_{\mathrm{m}}, \mathrm{MPa}$ & 0.80 & 0.52 & 0.69 & 0.78 \\
\hline \multirow[b]{2}{*}{ Mortero } & Flexión, $\mathrm{MPa}$ & 7.8 & 5.7 & 6.9 & 7.0 \\
\hline & $\begin{array}{l}\text { Compresión, } \\
\mathrm{MPa}\end{array}$ & 51.7 & 41.3 & 42.7 & 44.6 \\
\hline
\end{tabular}

Análisis de resultados de resistencia en prismas de albañilería

Los resultados de ensayo de prismas que muestra la Tabla 4, también dejan en evidencia que no hay grandes variaciones de resultados entre las distintas combinaciones conglomerantes y que en general las resistencias, aunque menores, no superan el 10\% menor respecto del hormigón con conglomerante $100 \%$ cemento, que es la mezcla de referencia o control. La norma NCh2123 (1997), indica que la resistencia básica de compresión de la albañilería $f_{\mathrm{m}}$ ', se determina del ensayo de cinco prismas coincidiendo con la expresión (1), con la que se han calculado valores de resistencia característica en este estudio. Esta norma también indica que cuando $f_{\mathrm{m}}$ ' no ha sido determinada por medio de ensayos de prismas, y tanto las unidades de albañilería como el mortero de junta cumplen con los requisitos de la norma, entonces $f_{\mathrm{m}}$ ' se puede determinar a partir del valor de resistencia de la unidad según la expresión siguiente:

$$
f_{\mathrm{m}}=0.30 f_{\mathrm{p}}
$$

Aplicando la expresión (3) al valor medio de resistencia de las unidades $\left(f_{\mathrm{p}}\right.$, referida al área bruta), para todas las combinaciones con puzolana, para la serie con ceniza volante y con polvo del desierto, se obtiene un valor de resistencia básica de compresión de prismas 3.9\% inferior al valor de resistencia característica prismática efectivamente medido a través del ensayo de cinco prismas, pero para la serie con ceniza volcánica, se obtiene un $2.1 \%$ superior al valor medido a través del ensayo de 5 prismas homólogos. Esto validaría lo especificado por la norma NCh1928 (1993) en (3), ya que indica que dicha expresión es adecuada para asumirla como valor $f_{\mathrm{m}}$, , lo que es un buen criterio. Sin embargo, esto no se cumple para hormigón $100 \%$ cemento, ya que la evaluación de (3) sobrevalora la resistencia básica de la compresión del prisma para asumirla como valor $f_{\mathrm{m}}$, en los procesos de cálculo estructural. De hecho, la valorización de (3), provee un valor de resistencia prismática superior en un $15.3 \%$ del valor de resistencia prismática medida a través del ensayo de cinco prismas. Este efecto se debe muy probablemente al endurecimiento o rigidez a temprana edad que produce el proceso de hidratación del cemento Portland en comparación a las mezclas con adiciones puzolánicas; condición de rigidez que se pierde con la esbeltez del prisma.

\section{Análisis de resultados de resistencia en muretes de allbañilería}

La norma NCh2123 (1997) indica que la resistencia básica 
de corte de la albañilería $\tau_{\mathrm{m}}$, se determina del ensayo de cinco muretes coincidiendo con la expresión (1) anterior. Esta norma también indica que cuando $\tau_{\mathrm{m}}$ no ha sido determinada por medio de ensayos de muretes, se pueden utilizar valores indicativos de resistencia básica de corte que esta norma la dispone en la Tabla 1 del ítem 5.7.2. En ella se indica que para el caso de unidades de bloque con resistencia $f_{\mathrm{p}} \geq 5.0 \mathrm{MPa}$ y grado del mortero $\geq \mathrm{M} 10$ (10 MPa), que sería el caso del presente estudio, el valor indicativo de resistencia básica de corte $\tau_{\mathrm{m}}$ será $0.30 \mathrm{MPa}$.

De la Tabla 4 se puede observar que los valores característicos de resistencia de corte $\tau_{\mathrm{m}}$ obtenidos experimentalmente de los ensayos de muretes, en el caso más desfavorable (conglomerante con ceniza volante), resultan un $73 \%$ mayor que el valor $0.30 \mathrm{MPa}$ indicados por norma (valor sin ensayo de muretes). Se concluye que estas mezclas con conglomerantes puzolánicos reportan muy buena resistencia de corte de la albañilería; que, para un país altamente sísmico como Chile, la resistencia de corte $\tau_{\mathrm{m}}$ de la albañilería debería ser el referente de calidad.

\section{Análisis de resultados de resistencia mecánica en mortero de junta}

Los valores de resistencia a compresión de mortero de junta que se muestran en la Tabla 4, dejan en evidencia los altos valores que se obtienen en toda mezcla conglomerante; muy por sobre la exigencia de las normas de albañilería que indican uso de mortero de junta igual o superior a M10 (10 $\mathrm{MPa}$ ). De todos los resultados de resistencia a compresión (a 28 días de edad) expuestos, resultan superiores los de $100 \%$ cemento respecto de los conglomerantes con adiciones puzolánicas, debido probablemente a que la reactividad de las puzolanas a dicha edad aún no se manifiesta del todo en la mezcla.

\section{Análisis de selectividad de mezclas conglomerantes puzolánicas para fabricar bloques}

Dado que todas las mezclas conglomerantes cumplen con exigencias de resistencia relacionadas con albañilería, la selección de la mezcla conglomerante se hace por comparación entre ellas a través de la evaluación del parámetro arbitrario $K$, ecuación (2), lo que se puede observar en la Tabla 5.
Tabla 5: Mezclas conglomerantes, evaluación del parámetro K

\begin{tabular}{|l|c|c|c|c|}
\cline { 2 - 5 } \multicolumn{1}{c|}{} & \multicolumn{4}{c|}{ Hormigón según conglomerante } \\
\hline Detalle & $\begin{array}{c}100 \% \\
\text { OPC }\end{array}$ & $\begin{array}{c}60 / 40 \\
\text { Ceniza } \\
\text { volante }\end{array}$ & $\begin{array}{c}70 / 30 \\
\text { Ceniza } \\
\text { volcánica }\end{array}$ & $\begin{array}{c}80 / 20 \\
\text { Polvo } \\
\text { desierto }\end{array}$ \\
\hline Costo unitario, \% & 100.0 & 18.0 & 14.0 & 10.0 \\
\hline $\begin{array}{l}\text { Costo mezcla } \\
\text { conglomerante, \% }\end{array}$ & 100.0 & 67.2 & 74.2 & 82.0 \\
\hline $\begin{array}{l}\text { Resistencia } \\
\text { prismática, MPa }\end{array}$ & 4.34 & 4.25 & 3.93 & 4.19 \\
\hline $\begin{array}{l}\text { Resistencia básica } \\
\text { de corte, MPa }\end{array}$ & 0.80 & 0.52 & 0.69 & 0.78 \\
\hline $\begin{array}{l}\text { K para resistencia } \\
\text { prismática }\end{array}$ & 4.34 & 6.32 & 5.30 & 5.11 \\
\hline $\begin{array}{l}K \text { para resistencia } \\
\text { básica de corte }\end{array}$ & 0.80 & 0.77 & 0.93 & 0.95 \\
\hline
\end{tabular}

Por medio de la evaluación del parámetro $K$, la Tabla 5 deja en evidencia lo conveniente que resulta utilizar combinaciones conglomerantes con puzolanas y que ante similar resistencia, el mayor beneficio de costo lo producirá la combinación conglomerante que mayor reduce el uso de cemento por su alto valor económico. En este sentido, si la referencia importante para un proyecto es la resistencia prismática, la combinación conglomerante que resulta con mayor valor del parámetro $K$, es la que utiliza ceniza volante, teniendo ventajas sobre cualquier otro tipo de mezcla. Sin embargo, si para un proyecto la referencia importante es la resistencia de corte, entonces la mejor combinación conglomerante resulta con uso de polvo del desierto ya que provee un valor mayor del parámetro $K$. Ambas situaciones, proveen suficiente resistencia mecánica (en su tipo) al más bajo costo económico.

\section{Conclusiones}

Las menores resistencias que muestran las probetas que utilizan la combinación de conglomerante con puzolana en comparación con probetas que utilizan $100 \%$ cemento Portland, se explican porque las puzolanas tienen una reactividad más lenta que el cemento Portland, dado que se activan por acción del hidróxido cálcico producido en la hidratación del cemento (Malhotra y Metha, 1996). Por esta razón y dado que hay estudios previos que avalan resultados de conglomerantes puzolánicos a 90 días (Roldán et al., 2013), es altamente probable que estas diferencias de resistencias a medida que se supere la edad de 28 días, disminuyan aún más o superen en resistencia a las probetas 
$100 \%$ cemento, lo que reafirmaría las bondades de utilizar este tipo de combinaciones conglomerantes en albañilería de bloques.

En general se cumple con los objetivos propuestos, que es factible el remplazo de cemento por materiales puzolánicos en albañilería de bloques, ya que se comprueba mediante resultados experimentales de resistencia mecánica, el cumplimiento con las normas vigentes en Chile. Además al disminuir la demanda de cemento, se ayuda a la sostenibilidad del ambiente al disminuir indirectamente la generación de $\mathrm{CO}_{2}$. También se debe considerar la reducción del costo económico utilizando productos naturales como ceniza volcánica y polvo del desierto y desecho industrial como ceniza volante.

La ceniza volante hoy es un pasivo industrial que no tiene costo de adquisición y tal vez las bondades de su uso como reemplazo de cemento que muestran estudios como este, con el tiempo puede cambiar esta condición. En cambio, tanto el polvo del desierto como la ceniza volcánica, representan bienes económicos libres, ya que uno se encuentra disponible en cualquier lugar del desierto del norte de Chile y el otro, disponible en ciertos lugares del país y en toda la Cordillera de los Andes. Por lo que, el aporte que hacen a la elaboración de mezclas cementantes no se pueden despreciar ya que contribuirían a un incremento de resistencia mayor que a una similar reducción del contenido de cemento en la mezcla y a una reducción no despreciable en el costo económico de edificación.

\section{Referencias}

Breitkreuz, C., de Silva, S.L., Wilke, H.G., Pfänder, J.A. and Renno, A.D. (2014). Neogene to Quaternary ash deposits in the Coastal Cordillera in northern Chile: Distal ashes from supereruptions in the Central Andes. Journal of Volcanology and Geothermal Research 269, 68-82.

Malhotra, V.M. and Metha, P.K. (1996). Pozzolanic and cementitious materials. Gordon and Breach Publishers, Ottawa, Canada

Metha, P.K. and Monteiro, P.J.M. (2006). Concrete: microstructure, properties, and materials. Third edition. McGraw-Hill. New York, USA

NCh158 (1967). Cementos - Ensayo de flexión y compresión de morteros de cemento. Instituto Nacional de Normalización INN, Santiago, Chile
NCh181 (1965). Bloques huecos de hormigón de cemento. Instituto Nacional de Normalización INN, Santiago, Chile

NCh181 (2006). Bloques de hormigón para uso estructural-Requisitos generales. Instituto Nacional de Normalización INN, Santiago, Chile

NCh182 (2008). Bloques de hormigón para uso estructural-Ensayos. Instituto Nacional de Normalización INN, Santiago, Chile

NCh1116 (2008). Áridos para morteros y hormigones-Determinación de la densidad aparente. Instituto Nacional de Normalización INN, Santiago, Chile

NCh1117 (2010). Áridos para morteros y hormigones-Determinación de las densidades real y neta y de la absorción de agua de las gravas. Instituto Nacional de Normalización INN, Santiago, Chile

NCh1239 (2009). Áridos para morteros y hormigones-Determinación de las densidades real y neta y de la absorción de agua de las arenas. Instituto Nacional de Normalización INN, Santiago, Chile

NCh1928 (1993). Mod-2009. Albañilería armada-Requisitos de diseño y cálculo. Instituto Nacional de Normalización INN, Santiago, Chile

NCh2123 (1997). Mod-2003. Albañilería confinada-Requisitos de diseño y cálculo. Instituto Nacional de Normalización INN, Santiago, Chile

O’Rourke, B., McNally, C. and Richardson, M.G. (2009). Development of calcium sulfate-ggbs-Portland cement binders. Construction and Building Materials 23(1), 340-346

Roldán, W. (2003). Construcción en albañilería de bloques de cemento y su aplicación en el norte de Chile. XIV Jornadas Chilenas del Hormigón. Universidad Austral de Chile, Valdivia, Chile

Roldán, W. y Robles, W. (2014). Factibilidad técnica de fabricación de hormigón con chuzca. Revista de Ingeniería Innova 7, 47-56

Roldán, W. y Pavéz, H. (2011). Factibilidad de elaboración de hormigón con cenizas volantes provenientes de centrales termoeléctricas de la región de Antofagasta. XVIII Jornadas Chilenas del Hormigón. Universidad Técnica Federico Santa María, Valparaíso, 78-92

Roldán, W., Zetola, V. y Robles, W. (2013). Conglomerantes especiales a base de materiales puzolánicos disponibles en la II Región de Chile. Revista de Ingeniería Innova 6, 87-97 